\title{
P35 Education Level may Modify the Association Between Cardiac Index and Cognitive Function Among Elders with Normal Ejection Function
}

\author{
Cheng Hao-Min ${ }^{1,2}$, Chuang Shao-Yuan ${ }^{3}$, Ko Yu-Ting, Liao Chao-Feng ${ }^{1}$, Pan Wen-Harn ${ }^{4}$, Liu Wen-Ling ${ }^{3}$, \\ Hung Chen-Ying ${ }^{5}$, Chen Chen-Huan ${ }^{2,3}$ \\ ${ }^{1}$ Faculty of Medicine, National Yang-Ming University School of Medicine, Taipei, Taiwan, R.O.C. \\ ${ }^{2}$ Department of Medical Education, Taipei Veterans General Hospital, Taipei, Taiwan, R.O.C. \\ ${ }^{3}$ Public Health Sciences Institute, National Health Research Institutes, Miaoli, Taiwan, R.O.C. \\ ${ }^{4}$ Institutes of Biomedical Sciences, Academia Sinica, Taipei, Taiwan, R.O.C. \\ ${ }^{5}$ Taipei Veterans Hospital, Hsinchu Branch, Hsinchu, Taiwan, R.O.C.
}

\section{ABSTRACT}

Background: Lower cardiac index (CI) in elders without clinical heart disease has been associated with impaired cognitive function and incident dementia. In the present study, we investigated the modulating effects of education level and arterial stiffness on the association between $\mathrm{CI}$ and cognitive function among older adults.

Methods: A total of 723 elders ( $\geq 60$ years, $50.1 \%$ female) with normal left ventricular ejection-fraction ( $\geq 50 \%)$ were identified from the Cardiovascular Diseases Risk Factor Two-Township Study. CI was calculated from the Doppler-derived stroke volume. We evaluated arterial stiffness by measuring carotid-femoral pulse wave velocity (CFPWV) and global cognitive function by using the Mini-Mental Short Examination (MMSE). Education level was determined by years of formal education.

Results: In linear regression analysis adjusting for age, sex, formal years of education and CFPWV, CI was significantly positively associated with MMSE (BETA $=0.3440 .130, p=0.0082$ ). In logistic regression analysis adjusting for age, sex, formal years of education and CFPWV, subjects with a CI 75 percentile had a significantly lower risk of low MMSE $(<26)(\mathrm{OR}=0.495,95 \% \mathrm{CI}=$ $0.274-0.896, p=0.02$ ). In subgroup analysis, higher CI was significantly associated with higher MMSE and lower risk of low MMSE only in elders with 9 years of formal education. Causal mediation analysis suggests that higher CI maintains higher MMSE in elders with lower education levels whereas higher CFPWV causes lower MMSE in all elders.

Conclusion: In elders with normal ejection fraction, a higher CI was associated with a lower risk of cognitive function impairment, independent of arterial stiffness, mainly in subjects with a lower education level and possibly a smaller cognitive reserve.

() 2019 Association for Research into Arterial Structure and Physiology. Publishing services by Atlantis Press International B.V. This is an open access article distributed under the CC BY-NC 4.0 license (http://creativecommons.org/licenses/by-nc/4.0/). 